\title{
Velocity vorticity-based large eddy simulation with the boundary element method
}

\author{
J. Ravnik, L. Škerget \& M. Hriberšek \\ University of Maribor, Faculty of Mechanical Engineering, \\ Maribor, Slovenia
}

\begin{abstract}
A large eddy simulation using velocity-vorticity formulation of the incompressible Navier-Stokes equations in combination with the turbulent heat transfer equation is proposed for the solution of the turbulent natural convection drive flow in a 1:4 enclosure. The system of equations is closed by an enstrophy based subgrid scale model. The Prandtl turbulent number is used to estimate turbulent diffusion in the heat transfer equation. The boundary element method is used to solve the kinematics equation and estimate the boundary vorticity values. The vorticity transport equation is solved by the finite element method. The numerical example investigated in the paper is the onset of a turbulent flow regime occurring at high Rayleigh number values $\left(R a=10^{7}-10^{10}\right)$. The formation of vortices in the boundary layer is observed, along with buoyancy driven diffusive convective transport. Quantitative comparison with the laminar flow model and the work of other authors is also presented in terms of Nusselt number value oscillations.
\end{abstract}

\section{Introduction}

Over the last few decades two-dimensional buoyancy driven flows have been investigated thoroughly by several authors. Natural convection in a rectangular enclosure is present in many industrial applications, such as the cooling of electronic circuitry, nuclear reactor insulation and ventilation of rooms.

A benchmark solution for two-dimensional flow of Boussinesq fluid in a square differentially heated enclosure was presented by De Vahl Davies [1]. They used the stream function-vorticity formulation. 2D DNS was preformed by Xin and Le Quéré [2] for an enclosure with aspect ratio 4 up to Rayleigh number based on the enclosure height $10^{10}$ using expansions in series of Chebyshev polynomials. 
Salat et al. [3] compared the results of modelling turbulent natural convection at high Rayleigh number between an experiment, 2D LES, 2D DNS and 3D LES computations. They reported that only minor differences are observed between the $2 \mathrm{D}$ and $3 \mathrm{D}$ results and concluded that a $2 \mathrm{D}$ calculation could be used as a first approximation for general flow structure in cavities at Rayleigh number about $10^{10}$.

In the present work we have studied the onset of natural convection in a $1: 4$ differentially heated enclosure within the incompressible Boussinesq approximation. Transition from two-dimensional steady laminar flow at enclosure width based Rayleigh number $R a=10^{6}$ via oscillatory motion at $R a=10^{7}, R a=10^{8}$ to chaotic (turbulent) fluid flow at $10^{9}, 10^{10}$ is simulated. The planar Large Eddy Simulation (LES) is used for velocity-vorticity formulation of the incompressible Navier-Stokes equations. The velocity vorticity formulation of the Navier-Stokes equations in combination with the boundary element method is a promising concept for numerical solution of fluid flow problems. Solution of the kinematics equation is obtained by the boundary element method (BEM) and provides boundary vorticity values and hence a well posed vorticity transfer equation. We propose the usage of BEM because of its unique advantage for solving the boundary. Unfortunately, solution of a Poisson type equation with BEM requires huge integral matrices, which poses computer storage problems and limits the maximum number of nodes. We have used a wavelet transform technique proposed by Ravnik et al. [4] to compress the matrices of integrals and thus decrease the storage requirements. The LES based vorticity transport equation is solved by the finite element method (FEM).

\section{$2 \vec{v}-\vec{\omega}$ LES}

Incompressible viscous fluid flow within the Boussinesq approximation is governed by the following system of equations. Mass conservation can be stated by

$$
\vec{\nabla} \cdot \vec{v}=0
$$

while conservation of momentum is

$$
\frac{\partial \vec{v}}{\partial t}+(\vec{v} \cdot \vec{\nabla}) \vec{v}=-\frac{R a}{P r R e^{2}} T \vec{g}-\frac{1}{E u} \vec{\nabla} p+\frac{1}{R e} \nabla^{2} \vec{v},
$$

and the energy equation is

$$
\frac{\partial T}{\partial t}+(\vec{v} \cdot \vec{\nabla}) T=\frac{1}{R e P r} \nabla^{2} T
$$

The system is fully defined by specifying the Euler, Reynolds, Prandtl and Rayleigh numbers. Vorticity is defined by $\vec{\omega}=\vec{\nabla} \times \vec{v}$ and is also divergence free. When 
introducing the velocity vorticity formulation (Škerget et al. [5]) one combines the velocity and vorticity into the kinematics equation

$$
\nabla^{2} \vec{v}+\vec{\nabla} \times \vec{\omega}=0
$$

which connects the velocity and vorticity fields at all points in space and time.

We define the filtering operation of an arbitrary function $u(\vec{x}, t)$ with the following convolution integral

$$
\bar{u}(\vec{x}, t)=\int_{\Omega} G(\vec{r}, \vec{x}) u(\vec{x}-\vec{r}, t) d \vec{r},
$$

where $G(\vec{r}, \vec{x})$ stands for the filter kernel, integration encompasses the whole domain $\Omega$. By choosing a homogenous filter kernel filtering commutes differentiation with respect to coordinate, thus the filtered kinematics equation is

$$
\overline{\nabla^{2} \vec{v}+\vec{\nabla} \times \vec{\omega}}=\nabla^{2} \overline{\vec{v}}+\vec{\nabla} \times \overline{\vec{\omega}}=0 .
$$

In order to derive the velocity vorticity based LES we will rewrite the transport equation for momentum (2) into a transport equation for vorticity and filter it. The advection term (second on the left hand side of (2)) may be rewritten as

$$
(\vec{v} \cdot \vec{\nabla}) \vec{v}=\frac{1}{2} \vec{\nabla} v^{2}-\vec{v} \times \vec{\omega} .
$$

We use (7) in equation (2) and rewrite the last term on the right hand side of (2) with the aid of the kinematics equation, arriving at

$$
\frac{\partial \vec{v}}{\partial t}+\frac{1}{2} \vec{\nabla} v^{2}-\vec{v} \times \vec{\omega}=-\frac{R a}{P r R e^{2}} T \vec{g}-\frac{1}{E u} \vec{\nabla} p-\frac{1}{R e} \nabla \times \vec{\omega} .
$$

When a curl of the whole equation (8) is taken, both gradient terms vanish. Thus pressure is eliminated from the equation. Bearing in mind the vorticity definition, we have

$$
\frac{\partial \vec{\omega}}{\partial t}-\vec{\nabla} \times(\vec{v} \times \vec{\omega})=-\frac{R a}{\operatorname{Pr} R e^{2}} \vec{\nabla} \times T \vec{g}-\frac{1}{R e} \vec{\nabla} \times \nabla \times \vec{\omega} .
$$

When equation (9) is filtered, commutation properties of the homogenous filter are used to write

$$
\frac{\partial \overline{\vec{\omega}}}{\partial t}-\vec{\nabla} \times \overline{(\vec{v} \times \vec{\omega})}=-\frac{R a}{P r R e^{2}} \vec{\nabla} \times \bar{T} \vec{g}-\frac{1}{R e} \vec{\nabla} \times \nabla \times \overline{\vec{\omega}} .
$$

The difference between the unfiltered (9) and the filtered (10) vorticity transport equation is in the nonlinear advection term. Filter of the vector product of velocity and vorticity fields is not equal to a vector product of filtered fields. Equation (10) will be rewritten in a form that is equivalent to the form of (9) with an additional term, which will account for the contribution of the filtered field. The additional, 
subgrid term will be modelled in such manner, that it will have a dissipative effect. Therefore we introduce the residual vorticity vector as the difference between the filter of the vector product of velocity and vorticity fields and a vector product of filtered fields $\vec{\tau}^{\omega}=\overline{\vec{v}} \times \vec{\omega}-\overline{\vec{v}} \times \overline{\vec{\omega}}$. With this, equation (10) may be rewritten

$$
\frac{\partial \overline{\vec{\omega}}}{\partial t}-\vec{\nabla} \times(\overline{\vec{v}} \times \overline{\vec{\omega}})=-\frac{R a}{\operatorname{Pr} R e^{2}} \vec{\nabla} \times \bar{T} \vec{g}-\frac{1}{R e} \vec{\nabla} \times \nabla \times \overline{\vec{\omega}}+\vec{\nabla} \times \vec{\tau}^{\omega} .
$$

According to the turbulent vorticity transfer theory of Taylor [6], the subgrid term $\vec{\nabla} \times \vec{\tau}^{\omega}$ describes the dissipation of vorticity due to subgrid scales. To derive the final form of the filtered vorticity transfer equation we make use of $\vec{\nabla} \times \vec{\nabla} \times \overline{\vec{\omega}}=$ $-\nabla^{2} \overline{\vec{\omega}}$ and $\vec{\nabla} \times(\overline{\vec{v}} \times \overline{\vec{\omega}})=(\overline{\vec{\omega}} \cdot \vec{\nabla}) \overline{\vec{v}}-(\overline{\vec{v}} \cdot \vec{\nabla}) \overline{\vec{\omega}}$ :

$$
\frac{\partial \overline{\vec{\omega}}}{\partial t}+(\overline{\vec{v}} \cdot \vec{\nabla}) \overline{\vec{\omega}}=(\overline{\vec{\omega}} \cdot \vec{\nabla}) \overline{\vec{v}}-\frac{R a}{{\operatorname{Pr} R e^{2}}^{2}} \vec{\nabla} \times \bar{T} \vec{g}+\frac{1}{\operatorname{Re}} \nabla^{2} \overline{\vec{\omega}}+\vec{\nabla} \times \vec{\tau}^{\omega} .
$$

The Stokes derivative of vorticity on the right hand side of equation (12) is equal to the twisting and stretching term, buoyancy, viscous diffusion and the subgrid term. The equation is nonlinear due to the product of velocity and vorticity, which are kinematically depended quantities. In two dimensions, only the component of vorticity that is perpendicular to the plan of flow is nonzero, thus vorticity may be regarded as a scalar quantity. The vortex twisting and stretching term vanishes is cases of planar flow. The buoyancy term, which includes the temperature, binds the vorticity transport equation to the energy equation.

Before filtering of the energy equation (3) we rewrite the advection term with $\vec{\nabla} \cdot(T \vec{v})=(\vec{v} \cdot \vec{\nabla}) T:$

$$
\frac{\partial \bar{T}}{\partial t}+\vec{\nabla} \cdot(\overline{T \vec{v}})=\frac{1}{\operatorname{RePr}} \nabla^{2} \bar{T}
$$

The nonlinear term is rewritten by introducing the residual temperature vector $\vec{\tau}^{h}=\overline{T \vec{v}}-\bar{T} \overline{\vec{v}}$. The final form of the filtered energy equation is

$$
\frac{\partial \bar{T}}{\partial t}+\vec{\nabla} \cdot(\bar{T} \overline{\vec{v}})=\frac{1}{\operatorname{RePr}} \nabla^{2} \bar{T}-\vec{\nabla} \cdot \vec{\tau}^{h} .
$$

With an analogy to molecular viscosity, which drains the energy of the flow, we will describe the residual vectors by introducing subgrid scale viscosity and diffusivity

$$
\vec{\tau}^{\omega}=-\nu_{s g s} \vec{\nabla} \times \overline{\vec{\omega}}, \quad \vec{\tau}^{h}=\alpha_{s g s} \vec{\nabla} \bar{T} .
$$

The subgrid scale viscosity was modelled by the Mansour et al. [7] model, which is based on the local enstrophy of the large scales

$$
\nu_{s g s}=(C \Delta)^{2} \sqrt{\vec{\omega} \cdot \vec{\omega}} .
$$

The filter width is $\Delta=\left(\Delta_{x} \Delta_{y} \Delta_{z}\right)^{\frac{1}{3}}$ in 3D and $\Delta=\left(\Delta_{x} \Delta_{y}\right)^{\frac{1}{2}}$ in 2D. In the vicinity of walls, the model constant $C$ will be damped with Piomelli and/or Van 
Driest damping function. Based on experimental findings on isotropic turbulence, the subgrid scale viscosity is comparable to subgrid scale diffusivity, their relationship being close to linear. The turbulent Prandtl number is used to connect the two: $\alpha_{s g s}=\frac{\nu_{s g s}}{P r_{t}}$.

\section{Numerical method}

Sufficiently dense meshes must be used in order for the LES simulation to be successful. BEM requires fully populated matrices of integrals. Their size grows rapidly with the number of grid points. In order to be able to obtain the solution we have made the following simplifications: (i) let the flow be planar, vorticity is a scalar, (ii) we shall use wavelet compressed BEM for the solution of the boundary with the kinematics equation only and (iii) use FEM for the transport equations. In the following we shall briefly describe the wavelet compressed BEM and give the computational algorithm at the end of this section. Detailed derivation and explanation of the numerical method may be found in Ravnik et al. [8].

Using BEM for the calculation of boundary vorticity values, one arrives at the following system of linear equations

$$
\left[D^{\Gamma}\right]\left\{\bar{\omega}^{\Gamma}\right\}=([C]+[H])\left\{\bar{v}_{t}\right\}+\left[H^{t}\right]\left\{\bar{v}_{n}\right\}-\left[D^{\Omega \backslash \Gamma}\right]\left\{\bar{\omega}^{\Omega \backslash \Gamma}\right\}
$$

where matrices of integrals $\left[D^{\Gamma}\right],[C],[H]$ and $\left[H^{t}\right]$ are square, full, non-symmetric and have number of boundary nodes rows and columns. Although full, the these matrices do not require a lot of storage, since they are for boundary only. On the other hand, the matrix $\left[D^{\Omega \backslash \Gamma}\right]$ is rectangular and also full and non-symmetric. It has number of boundary nodes rows and number of internal nodes columns. In the discretized system of equations it must be multiplied with a vector of internal vorticity values $\left\{\bar{\omega}^{\Omega \backslash \Gamma}\right\}$ to form the right hand side of the system of equations.

The matrix vector product $\left[D^{\Omega \backslash \Gamma}\right]\left\{\bar{\omega}^{\Omega \backslash \Gamma}\right\}$ will be calculated with the aid of wavelet compression. The matrix will be compressed written in compressed row storage format and thus require less storage.

Let $W$ be the discrete wavelet transform matrix for vectors of arbitrary length introduced in Ravnik et al. [4]. Since the product of the wavelet matrix with its transpose is the identity matrix, we may write

$$
\left[D^{\Omega \backslash \Gamma}\right]\left\{\bar{\omega}^{\Omega \backslash \Gamma}\right\}=W^{T}(\underbrace{W\left[D^{\Omega \backslash \Gamma}\right] W^{T}}_{\left[D_{W}^{\Omega \backslash \Gamma}\right]} W\left\{\bar{\omega}^{\Omega \backslash \Gamma}\right\}) .
$$

The wavelet compressed matrix of integrals $\left[D_{W}^{\Omega \backslash \Gamma}\right]=W\left[D^{\Omega \backslash \Gamma}\right] W^{T}$ is calculated only once, prior to the start of the iterative process. In absolute sense small matrix elements are thresholded and compressed row storage format is used for remaining nonzero elements.

A parallel code has been written to perform the wavelet compression, since the full matrix does not fit into single computer memory. Clusters of single processor 
nodes have been used. Communication was preformed via MPI. During compression, multiplication with random test vectors is done to establish the relative error $\epsilon$ of the matrix vector product.

Without further details, the computational algorithm may be summarised as follows: Solve kinematics equation for boundary vorticity values using internal vorticities from previous nonlinear iteration step using wavelet compressed BEM. Solve the kinematics equation again for domain velocities by FEM, using new boundary vorticities obtained from BEM in the previous step to form right hand side vector. Solve the energy equation to obtain the new temperature field by FEM. Solve vorticity transport equation for domain vorticities by FEM, using the new velocity field and use boundary vorticities from kinematics as boundary conditions. Use under-relaxation for computing new domain vorticity values and loop until convergence is achieved.

\section{Validation}

Using wavelet compression in the numerical algorithm introduces an error. In order to estimate the largest compression ratio, that does not effect the accuracy of a high $R e$ computation, we have preformed a standard lid driven cavity benchmark test at $R e=10^{4}$. Results were compared with Ghia et al. [9] benchmark. Table 1 gives different compression rations tested, while Figure 1 presents the results. We have found, that the solution obtained is virtually identical for $\epsilon=10^{-6}$ and $\epsilon=10^{-5}$ and differs only slightly for lower relative error values. Therefore, we decided that the limit $\epsilon=10^{-5}$, will be used for compression. The test also showed, that increasing mesh density enables higher compression for the same accuracy.

Table 1: Wavelet compression of domain integrals matrix on a square mesh with $182 \times 180$ nine node Lagrange elements. Total number of nodes in the mesh is 131765 . Number of all elements in the matrix is 188699016 .

\begin{tabular}{|c|c|}
\hline $\begin{array}{c}\text { share of } \\
\text { thresholded elements }\end{array}$ & $\begin{array}{c}\text { relative error } \\
\text { of multiplication } \epsilon\end{array}$ \\
\hline 0.7652 & $1.4 \cdot 10^{-6}$ \\
0.9217 & $1.0 \cdot 10^{-5}$ \\
0.9761 & $1.0 \cdot 10^{-4}$ \\
0.9870 & $1.2 \cdot 10^{-3}$ \\
\hline
\end{tabular}

\section{Differentially heated enclosure}

We consider an enclosure with width to height ratio of $1: 4$. The left vertical wall is heated, the right vertical wall is cooled, both are kept at constant temperatures. 
The Rayleigh number is defined by the temperature difference and the enclosure width. The top and bottom horizontal walls are adiabatic. The no-slip velocity boundary condition is employed on all solid walls.
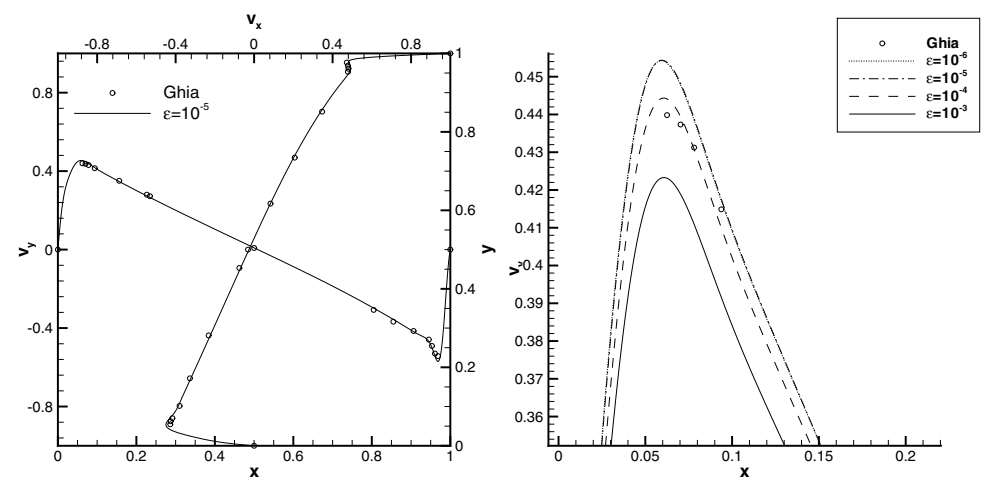

Figure 1: Comparison of velocity profiles in a lid driven cavity at $R e=10^{4}$ for different compression ratios with the benchmark solution of Ghia et al. [9].

The enclosure is filed with air $(\operatorname{Pr}=0.71)$, the subgrid scale constant was $C=0.1$, the turbulent Prandtl number was set to $P r_{t}=0.6$. Two meshes were used in simulations: up to Rayleigh number $R a \leq 10^{9}$ a mesh with $128 \times 200$ nine node Lagrange elements with approximately $10^{5}$ nodes and at Rayleigh number $10^{10}$ a $170 \times 300$ mesh with $2 \cdot 10^{5}$ nodes.

Steady state temperature field for $R a=10^{6}$ and averaged temperature fields at $R a=10^{7}$ without subgrid scale model and LES with Piomelli damping for $R a=10^{8}, R a=10^{9}$ and $R a=10^{10}$ are shown in Figure 2. While at $R a=10^{6}$ steady state is reached, at $R a=10^{7}$ the boundary layer becomes unstable and vortices are formed along the top of the hot wall and along the bottom part of the cold wall. Eddies are transported by convection up the hot wall and down the cold wall thus mixing the top and bottom parts of the enclosure. In the central part the temperature field is stratified and the flow virtually steady. The whole flow field is oscillatory and symmetric. At $R a=10^{8}$ the eddies are formed more frequently. The formation takes place in the top half of the hot wall and in the bottom half of the cold wall. The stratified central core becomes smaller, but still exists. The flow field is no longer symmetric (although the initial $R a=10^{6}$ flow field was) length scales of the structures in the flow are becoming smaller. There is no difference between calculations with and without the subgrid scale model. At $R a=10^{9}$ eddies are formed along the whole length of both vertical walls, most of them being formed at mid height. The central core is now thoroughly mixed and one can no longer speak of temperature stratification. The flow field includes eddies of various scales and is non-repeating, irregular and chaotic. At $R a=10^{10}$ the whole flow field is turbulent. The difference between calculation with and without 
subgrid scale model is evident at this Rayleigh number. Comparing heat transfer with benchmark proved that the LES simulation give the correct results.
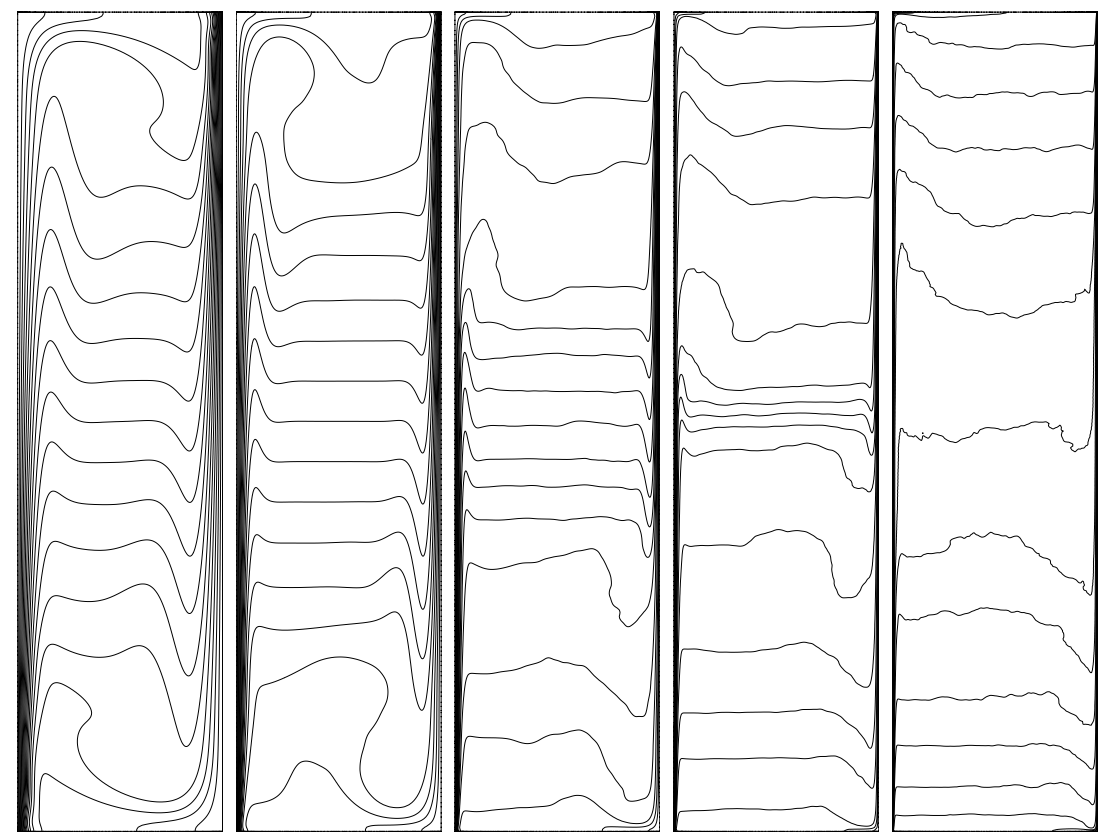

Figure 2: Temperature fields (1(0.05)0): left to right: steady state at $R a=10^{6}$, time averaged field at $R a=10^{7}$ without a subgrid scale model, time averaged LES field with damped subgrid scale model for $R a=10^{8}$, $R a=10^{9}$ and $R a=10^{10}$.

The heat transfer through the walls is represented by the average Nusselt number value, defined for our geometry by $N u=\frac{1}{H} \int_{0}^{H} \frac{\partial T}{\partial x} d y$. The Nusselt number versus time graphs are shown on Figure 3. The average values are compared with benchmark results of Xin and Le Quéré [2] in Table 2. Very good agreement is obtained.

\section{Conclusions}

The velocity vorticity formulation of LES in combination with the wavelet transform based boundary element method presented in this paper shows good potential for solving turbulent fluid flow problems with the large eddy simulation approach. Solution of boundary vorticity values with wavelet based BEM provides boundary conditions for the transport equations, which we are solving by FEM. Using the wavelet transform with the boundary element method enabled us to use meshes 

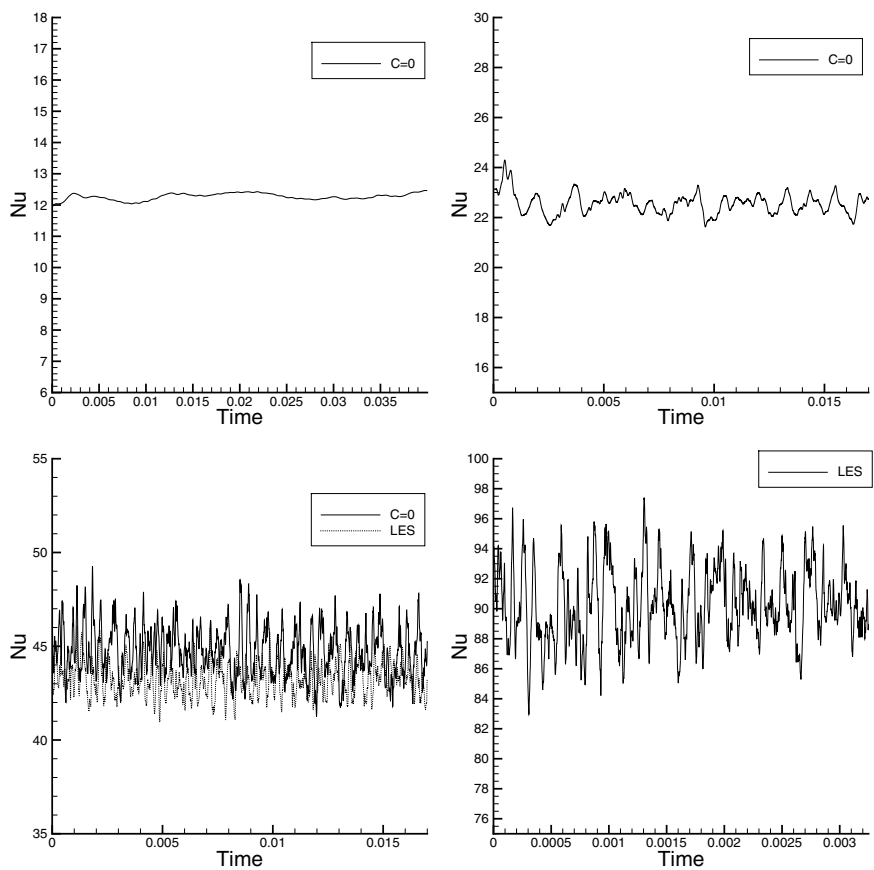

Figure 3: Time traces of Nusselt number for: $R a=10^{7}$ (upper left), $R a=10^{8}$ (upper right), $R a=10^{9}$ (lower left) in $R a=10^{10}$ (lower right).

Table 2: Average Nusselt number $N u$ and correlation comparison with benchmark DNS results of Xin et al. [2]. Piomelli damped $\left(L E S_{p}\right)$ as well as Van Driest damped $\left(L E S_{v d}\right)$ results are presented. (*) For $N u$ at $R a=10^{8}$ and $R a=10^{9}$ Xin's values were predicted using their $N u / R a^{1 / 4}$ relationship. (+) $N u$ prediction using our $N u / R a^{1 / 4}$ relationship.

\begin{tabular}{|c|c|c|c|c|c|c|}
\hline & \multicolumn{5}{|c|}{$N u$} & \multicolumn{2}{c|}{$N u / R a^{1 / 4}$} \\
\hline$R a$ & $C=0$ & $L E S_{p}$ & $L E S_{v d}$ & Xin [2] & present & Xin [2] \\
\hline $10^{7}$ & 12.27 & & & 12.3 & 0.2181 & 0.2185 \\
$3.125 \cdot 10^{7}$ & & $16.91^{+}$ & & 16.62 & & \\
$10^{8}$ & 22.5 & 22.5 & & $22.56^{*}$ & 0.2256 & \\
$1.56 \cdot 10^{8}$ & & $25.57^{+}$ & & 25.25 & & 0.2256 \\
$10^{9}$ & 44.77 & 43.25 & 43.67 & $43.63^{*}$ & 0.2432 & \\
$10^{10}$ & & & 90.25 & & & \\
\hline
\end{tabular}


with $2 \cdot 10^{5}$ nodes. Higher mesh densities will be possible in the near future, as well as the extension of the code to $3 \mathrm{D}$, by the use of domain decomposition and parallel computing.

\section{Acknowledgements}

The first author gratefully acknowledges the support of the parallel computer centres HLRS and CINECA in the framework of the EC-funded HPC-Europa project under contract number 506079.

\section{References}

[1] Davies, G.D.V., Natural convection of air in a square cavity: a bench mark numerical solution. Int J Numer Meth Fl, 3, pp. 249-264, 1983.

[2] Xin, S. \& Le Quéré, P., Direct numerical simulations of two-dimensional chaotic natural convection in a differentially heated cavity of aspect ratio 4 . J Fluid Mech, 304, pp. 87 - 118, 1995.

[3] Salat, J., Xin, S., Joubert, P., Sergent, A., Penot, F. \& Le Quéré, P., Experimental and numerical investigation of turbulent natural convection in a large air-filled cavity. Int J Heat Fluid Fl, 25, pp. 824-832, 2004.

[4] Ravnik, J., Škerget, L. \& Hriberšek, M., The wavelet transform for BEM computational fluid dynamics. Eng Anal Bound Elem, 28, pp. 1303-1314, 2004.

[5] Škerget, L., Alujeviè, A., Brebbia, C.A. \& Kuhn, G., Topics in Boundary Element Research, Springer-Verlag: Berlin, volume 5, chapter Natural and Forced Convection Simulation Using the Velocity-Vorticity Approach, 1989.

[6] Taylor, G.I., The transport of vorticity and heat through fluids in turbulent motion. Proc R Soc London Ser A, 135, pp. 685-705, 1932.

[7] Mansour, N.N., Ferziger, J.H. \& Reynolds, W.C., Large-eddy simulation of a turbulent mixing layer. Report TF-11, Thermosciences Div., Dept. of Mech. Eng., Standford University., 1978.

[8] Ravnik, J., Škerget, L. \& Hriberšek, M., 2D $\vec{v}-\vec{\omega}$ LES for the solution of natural convection in a differentially heated enclosure by wavelet transform based BEM and FEM. Eng Anal Bound Elem, submitted.

[9] Ghia, U., Ghia, K. \& Shin, C., High-Re solutions for incompressible flow using the Navier-Stokes equations and a multigrid method. J Comput Phys, 48, pp. 387-411, 1982. 